Mathematical Modelling and Analysis

Volume 17 Number 5, November 2012, 732-748

http://dx.doi.org/10.3846/13926292.2012.736416

(c) Vilnius Gediminas Technical University, 2012
Publisher: Taylor\&Francis and VGTU

http://www.tandfonline.com/TMMA

Print ISSN: 1392-6292

Online ISSN: 1648-3510

\title{
Comparison of Adaptive Meshes for a Singularly Perturbed Reaction-Diffusion Problem
}

\section{Andrej Bugajev and Raimondas Čiegis}

Vilnius Gediminas Technical University

Sauletekio av. 11, LT-10223 Vilnius, Lithuania

E-mail(corresp.): anb@vgtu. It

E-mail: rc@vgtu. It

Received September 7, 2012; revised September 28, 2012; published online November 1, 2012

\begin{abstract}
We consider a singular second-order boundary value problem. The differential problem is approximated by the Galerkin finite element scheme. The main goal is to compare the well known apriori Bakhvalov and Shishkin meshes with the adaptive mesh based on the aposteriori dual error estimators. Results of numerical experiments are presented.
\end{abstract}

Keywords: singularly perturbed boundary value problem, Galerkin method, adaptive meshes, a posteriori error estimates, Shishkin mesh, Bakhvalov mesh.

AMS Subject Classification: 65L10; 65L12; 65L50.

\section{Problem Formulation}

We consider the one-dimensional singularly perturbed boundary value problem

$$
\left\{\begin{array}{l}
-\varepsilon^{2} u^{\prime \prime}+q(x) u=f(x), \quad x \in(0,1), \\
u(0)=0, \quad u(1)=0,
\end{array}\right.
$$

where $f=f(x), q(x) \geq a>0$ are given sufficiently smooth functions, $0<$ $\varepsilon \ll 1$. It is well-known that solutions of this problem can have steep exponential boundary layers, therefore a numerical approximation of singularly perturbed problems is a challenging task. A good review on the state of art in this field is given in $[13,19]$. Two general strategies can be used to construct efficient and uniformly convergent numerical algorithms for problems with boundary layers. The first one is based on special types of finite difference or finite volume approximations. Here we mention exponentially fitted finite difference and finite element schemes [9, 11, 20, 22, 23]. Exponentially fitted finite difference schemes can be obtained approximating coefficients of the differential equation by piece-wise constant functions and solving exactly 
the obtained perturbed differential equations. In finite element algorithms the Petrov-Galerkin method is used by upwinding the test functions.

The second strategy uses standard finite difference or finite element discretizations on non-uniform meshes. The main idea is to resolve exponential boundary layers by a suitable mesh. Starting from the paper of Bakhvalov [4], efficient numerical algorithms were constructed using special adaptive meshes. These meshes are exponentially stretched within the boundary layers. In [21], Shishkin proposed a simpler type of adaptive meshes, which can be constructed a priori as a function of the singular parameter $\varepsilon$ and are piecewise-uniform. It was proved that such meshes can be used for wide classes of singularly perturbed problems.

Roos and Linß have proposed simple sufficient conditions to prove a uniform convergence on layer-adapted meshes [18]. They have applied these conditions not only for the Bakhvalov and Shishkin meshes, but also have analyzed discrete schemes proposed by Vulanovic [24, 25], Boglaev [7]. The Bakhvalov and Shishkin meshes were compared in [25], where a quasi-linear singularly perturbed boundary value problem was considered and the Shishkin grid was generalized and improved.

The other important trend of theoretical analysis deals with a posteriori error estimates for the numerical solutions of singularly perturbed elliptic equations. Such computable estimates for singularly perturbed or convectiondominated problems where investigated, e.g. in $[2,3,16,17]$. These estimates can be used to construct non-uniform adaptive meshes by using the equidistribution of a computed approximation of the error monitoring function. The benchmark adaptive meshes can be constructed by the equidistribution of monitoring functions that are based on the exact solution (see, [17] for convection dominated test problems).

For convection-diffusion equations different a posteriori error estimators were considered by John [12], including gradient indicator, residual based error estimators for different norms and two error estimators which are defined by solution of local Neumann problems. The accuracy of numerical approximations was investigated on adaptive meshes. The obtained results show that none of the considered error estimators work robustly in all tests. In [6], the finite difference approximation of a model singularly perturbed reaction-diffusion boundary value problem (1.1) is investigated. The meshes are based on the equidistribution of a positive monitoring function that takes into account a power of the second derivative of the solution.

Reliable a posteriori estimators for the error in $H^{1}$ norm for a $2 \mathrm{D}$ singularly perturbed reaction-diffusion model were constructed and investigated in [14]. Vulanovic [26] analyzed the relationship between layer-resolving transformations and mesh generating functions for numerical solution of singularly perturbed boundary value problems. Linß [15] considered non-monotone FEM discretization for singularly perturbed problem (1.1). He proved a priori and a posteriori error bounds in the maximum norm.

Non-local boundary conditions are also considered for singularly perturbed diffusion-reaction problems. Exponentially fitted schemes were used to approximate the problem with three-point nonlocal condition by Amiraliyev and 
Cakir [1]. For integral nonlocal conditions such schemes were investigated by Čiegis [9], Cakir and Amiraliyev [8].

Our goal is to investigate the optimality of the Bakhvalov and Shishkin meshes for singularly perturbed diffusion-reaction problems. We compare these meshes with the adaptive meshes constructed by using general duality-based a priori error estimators in the $L_{2}$ and energy norms [5, 10]. A standard technique of the equidistribution of a computed error monitoring function is applied in order to construct optimal meshes for different norms. It is wellknown that only the uniform norm enables us to resolve boundary layers for general meshes. The usage of integral $L_{2}$ and energy norms is justified for adaptive meshes, which can be seen as special layer-resolving transformations.

The rest of the paper is organized as follows. In Section 2, the Galerkin method is presented. The mesh generation routines are developed in Section 3. In Section 4, we compare different meshes and results of numerical experiments are presented. Some final conclusions are presented in Section 5.

\section{The Galerkin Finite Element Method}

Let us define the test functions space

$$
V=\left\{v: \int_{\Omega}\left(\left|v^{\prime}\right|^{2}+v^{2}\right) d x<\infty, v(0)=v(1)=0\right\}
$$

and we take the trial functions space $V^{1}=V$. We apply the standard Galerkin method to problem (1.1) and integrate the differential equation by parts, this gives the variational problem formulation: find $u \in V$ such that

$$
\int_{0}^{1}\left(\varepsilon^{2} u^{\prime} v^{\prime}+q u v\right) d x=\int_{0}^{1} f v d x \quad \forall v \in V .
$$

We consider piecewise linear trial and test functions. Let $W_{h}: 0=x_{0}<x_{1}<$ $\cdots<x_{N-1}<x_{N}=1$ be a partition of $[0,1]$ and let $V_{h} \subset V$ be the corresponding finite element space of continuous piecewise linear functions. The finite element method is obtained by applying the Galerkin method to (2.1) with the finite dimensional space $V_{h} \subset V$ replacing $V$ and reads: find $U \in V_{h}$ such that

$$
\int_{0}^{1}\left(\varepsilon^{2} U^{\prime} v^{\prime}+q U v\right) d x=\int_{0}^{1} f v d x \quad \forall v \in V_{h} .
$$

Let $\varphi_{i}$ be nodal basis functions

$$
\varphi_{i}= \begin{cases}\left(x-x_{i-1}\right) / h_{i-0.5}, & x \in\left[x_{i-1}, x_{i}\right), \\ \left(x_{i+1}-x\right) / h_{i+0.5}, & x \in\left[x_{i}, x_{i+1}\right] \\ 0, & \text { otherwise }\end{cases}
$$


where $h_{j-0.5}=x_{j}-x_{j-1}$, then any $v \in V_{h}$ can be written as $v(x)=$ $\sum_{j=1}^{N-1} \mu_{j} \varphi_{j}(x)$, where $\mu_{j}$ are coefficients. We write $U$ in terms of nodal basis functions $\varphi_{j}, j=1, \ldots N-1$,

$$
U(x)=\sum_{j=1}^{N-1} U_{j} \varphi_{j}(x),
$$

and substitute this expression into (2.2). Choosing $v$ in (2.2) to be $\varphi_{i}, i=$ $1, \ldots, N-1$, we obtain the system of equations for $U_{j}$ which is tridiagonal. Solving this system gives $U(x)$ from $(2.3)$.

\section{Mesh Generation Using Duality-Based Technique}

In this section, we apply the well-known duality-based error estimates for the problem (1.1). A standard technique described e.g. in [10] for general second order boundary value problems is used. We make no attempt to construct special uniform in small parameter $\varepsilon$ error estimates, since boundary layers should be resolved by special layer-resolving transformations based on adaptive meshes. The error is estimated in the $L_{2}$ and energy norms.

\subsection{Error estimation in the $L_{2}$ norm}

Let us define $A u:=-\varepsilon^{2} u^{\prime \prime}+q u$, then problem (1.1) can be written as

$$
\left\{\begin{array}{l}
A u=f, \quad \text { in } x \in(0,1), \\
u(0)=0, \quad u(1)=0 .
\end{array}\right.
$$

A scalar product and the $L_{2}$ norm are defined as

$$
(u, v)=\int_{0}^{1} u v d x, \quad\|u\|=\sqrt{(u, u)} .
$$

Let us denote the error $e=u-U$. In order to estimate this error, we find $z$ as a solution of the dual problem

$$
\left\{\begin{array}{l}
A^{*} z=e, \quad \text { in } x \in[0,1] \\
z(0)=0, \quad z(1)=0 .
\end{array}\right.
$$

In our case $A^{*}=A$. Multiplying (3.1) by $e$, integrating by parts and considering $v=\pi_{h} z$ in $(2.2)$, where $\pi_{h} z \in V_{h}$ is the piecewise linear interpolant of $z$, we get the estimate

$$
(e, e) \leq\left\|h^{2}(q U-f)\right\|\left\|h^{-2}\left(z-\pi_{h} z\right)\right\| .
$$

Here $h=h(x)=x_{i}-x_{i-1}$ in $x_{i-1}<x \leq x_{i}$. The error of the the piecewise linear interpolant $\pi_{h} z$ can be estimated as [10]

$$
\left\|h^{-2}\left(z-\pi_{h} z\right)\right\| \leq C_{i}\left\|z^{\prime \prime}\right\|
$$


To estimate the term $\left\|z^{\prime \prime}\right\|$ in (3.3) we consider the following problem

$$
\left\{\begin{array}{l}
A w=\psi, \quad \text { in } x \in[0,1] \\
w(0)=0, \quad w(1)=0
\end{array}\right.
$$

where $\psi=\psi(x) \in V$ are functions that represent all possible error functions $e=e(x)$. Then the strong stability factor $S$ is defined by

$$
S=\max _{\psi \in V} \frac{\left\|w^{\prime \prime}\right\|}{\|\psi\|} .
$$

Consider there $\psi=e$, then from (3.1) we get that $w=z$ and obtain the estimate

$$
\left\|z^{\prime \prime}\right\| \leq S\|e\|
$$

Finally, from (3.2), (3.3) and (3.5) we obtain the aposteriori error estimate in the $L_{2}$ norm

$$
\|e\| \leq \bar{C}_{i}\left\|h^{2} q(U-f)\right\| .
$$

Here $\bar{C}_{i}=S C_{i}$ is a constant and it has no effect on construction of adaptive meshes.

\subsection{Error estimation in the energy norm}

Let us define the energy norm $\left\|e^{\prime}\right\|=\sqrt{\left(e^{\prime}, e^{\prime}\right)}$. Replacing $v \in V$ with an error function $e \in V$ in variational problem (2.1), considering $v=\pi_{h} e$ in (2.2), where $\pi_{h} e \in V_{h}$ is a piecewise linear interpolant of $e$, integrating by parts and using the Cauchy inequality, gives

$$
\varepsilon^{2}\left\|e^{\prime}\right\|^{2} \leq\|h(q U-f)\|\left\|h^{-1}\left(e-\pi_{h} e\right)\right\| .
$$

The interpolation error can be estimated as [10]

$$
\left\|h^{-1}\left(e-\pi_{h} e\right)\right\| \leq \widetilde{C}_{i}\left\|e^{\prime}\right\|,
$$

where $\widetilde{C}_{i}$ is a constant. Using this inequality in (3.7) we get an aposteriori error estimate in the energy norm

$$
\left\|e^{\prime}\right\| \leq \frac{\widetilde{C}_{i}}{\varepsilon^{2}}\|h(q U-f)\| .
$$

We note that in both aposteriori error estimates (3.6) and (3.8) the residual $R(U)=q U-f$ of the FE scheme multiplied by $h^{p}$ defines the contribution to the total error from the element $\left[x_{j-1}, x_{j}\right]$.

\subsection{Adaptive mesh generation}

The adaptive mesh is obtained by solving the minimization problem

$$
\min _{x_{1}, \ldots, x_{N-1}} \sum_{j=1}^{N} r_{j-0.5}, \quad \sum_{j=1}^{N} h_{j-0.5}=1,
$$


where $r_{j-0.5}$ is a local error estimate on the interval $\left[x_{j-1}, x_{j}\right]$. To minimize the total error the standard local error equidistribution technique is applied for a given monitoring function [10]. It reads: find $h_{j-0.5}, j=1, \ldots, N$, such that $r_{j-0.5}=$ const $, j=1, \ldots, N$.

Using aposteriori error estimates (3.6), (3.8) we get the following error equidistribution problem

$$
r_{j-0.5}=h_{j-0,5}^{2 p} \int_{x_{j-1}}^{x_{j}}(q U-f)^{2} d x=\text { const }, \quad j=1,2, \ldots, N,
$$

here $p=1$ for the energy norm and $p=2$ for the $L_{2}$ norm, respectively. Problem (3.10) can be solved by the inverse interpolation method.

\section{Comparison with Apriori Adaptive Meshes}

In this section we compare the adaptive mesh based on aposteriori error estimates with the well known Shishkin and Bakhvalov meshes.

\subsection{Comparison with the Bakhvalov mesh}

The Bakhvalov mesh [4] is generated by equidistributing

$$
\int_{x_{j-1}}^{x_{j}} M_{B}(x) d x=\text { const }, \quad j=1,2, \ldots, N
$$

the monitoring function

$$
M_{B}(x)=\max \left\{\alpha, e^{\left(-\frac{\rho x}{\sigma \varepsilon}\right)}, e^{\left(-\frac{\rho(1-x)}{\sigma \varepsilon}\right)}\right\}
$$

where $\varrho=\min _{x \in[0,1]} \sqrt{q(x)}, \sigma$ is a freely defined parameter, and $\alpha$ is a regularization parameter.

The information on function $f$ is not used in the Bakhvalov mesh. Also, we note, that in the Bakhvalov mesh the function $q(x)$ is approximated by the constant $\varrho=\min _{x \in[0,1]} \sqrt{q(x)}$.

In order to find a relation between duality-based a posteriori mesh and the Bakhvalov mesh we consider the problem

$$
\left\{\begin{array}{l}
-\varepsilon^{2} u^{\prime \prime}+\rho^{2} u=\bar{f}, \quad \text { in } x \in(0,1), \\
u(0)=0, \quad u(1)=0
\end{array}\right.
$$

where $\bar{f}=$ const. To obtain an analytical formula of the adaptive meshes in the $L_{2}$ and energy norms we use the exact solution of (4.3) instead of the Galerkin approximation (3.10):

$$
u(x)=\frac{\bar{f}}{\rho^{2}} \frac{-e^{\left(\frac{\rho x}{\varepsilon}\right)}+e^{\left(-\frac{\rho x}{\varepsilon}\right)}-e^{\left(\frac{\rho(1-x)}{\varepsilon}\right)}+e^{\left(-\frac{\rho(1-x)}{\varepsilon}\right)}-e^{\left(-\frac{\rho}{\varepsilon}\right)}+e^{\left(\frac{\rho}{\varepsilon}\right)}}{e^{\left(\frac{\rho}{\varepsilon}\right)}-e^{\left(-\frac{\rho}{\varepsilon}\right)}} .
$$


Then the residual of the Galerkin scheme can be computed as

$$
R(u)=\frac{\bar{f}}{e^{\left(\frac{\rho}{\varepsilon}\right)}-e^{\left(-\frac{\rho}{\varepsilon}\right)}}\left(-e^{\left(\frac{\rho x}{\varepsilon}\right)}+e^{\left(-\frac{\rho x}{\varepsilon}\right)}-e^{\left(\frac{\rho(1-x)}{\varepsilon}\right)}+e^{\left(-\frac{\rho(1-x)}{\varepsilon}\right)}\right) .
$$

We are interested to analyze the adaptive mesh only in the neighbourhood of boundary layers $0 \leq x \leq c \varepsilon / \rho$ and $0 \leq 1-x \leq c \varepsilon / \rho$, thus it is sufficient to take the following asymptotical approximation of the residual

$$
\widetilde{R}(u)=-\bar{f}\left(e^{-\frac{\rho x}{\varepsilon}}+e^{-\frac{\rho(1-x)}{\varepsilon}}\right) .
$$

Then from (3.10) we get the following error equidistribution problem

$$
h_{j-0,5}^{2 p} \int_{x_{j-1}}^{x_{j}}\left(e^{-\frac{\rho x}{\varepsilon}}+e^{-\frac{\rho(1-x)}{\varepsilon}}\right)^{2} d x=\text { const }, \quad j=1,2, \ldots, N .
$$

First, let us consider a left boundary layer at $x=0$. Since $\varepsilon \ll 1$, equations (4.5) can be simplified to

$$
h_{j-0,5}^{2 p} \int_{x_{j-1}}^{x_{j}} e^{-2 \rho x / \varepsilon} d x=\text { const }, \quad j=1,2, \ldots, N / 2 .
$$

Applying the standard midpoint rule for numerical integration after simple computations we derive the mesh equidistribution equations in the following form

$$
\int_{x_{j-1}}^{x_{j}} e^{-\frac{2 \rho x}{\varepsilon(2 p+1)}} d x=\text { const }, \quad j=1,2, \ldots, N / 2 .
$$

A boundary layer at $x=1$ is considered similarly. Thus the duality based adaptive mesh can be obtained by using the monitoring function

$$
M(x)= \begin{cases}e^{-\frac{2 \rho x}{\varepsilon(2 p+1)}}, & \text { in } x \in[0,0.5), \\ e^{\frac{2 \rho(x-1)}{\varepsilon(2 p+1)}}, & \text { in } x \in[0.5,1] .\end{cases}
$$

By taking $\sigma=\frac{2 p+1}{2}$ in (4.7) we get the monitoring function (4.2), which was used to construct the Bakhvalov mesh. In addition we have derived a rule to define parameter $\sigma$ for the Bakhvalov mesh: $\sigma=\frac{5}{2}$, if the global error is estimated in the $L_{2}$ norm, and $\sigma=\frac{3}{2}$, if the energy norm is used.

At the end of this subsection we present explicit formulas for the adaptive mesh, generated by the monitoring function (4.7). From (4.6) we get a system of nonlinear equations

$$
\int_{0}^{x_{j}} e^{\left(-\frac{\rho x}{\sigma \varepsilon}\right)} d x=\frac{2 j}{N} \int_{0}^{1 / 2} e^{-\left(\frac{\rho x}{\sigma \varepsilon}\right)} d x, \quad j=1, \ldots, N / 2,
$$




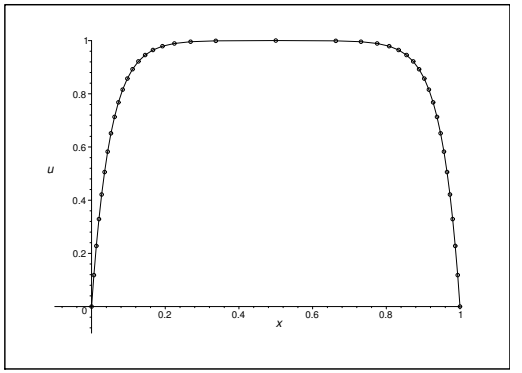

a)

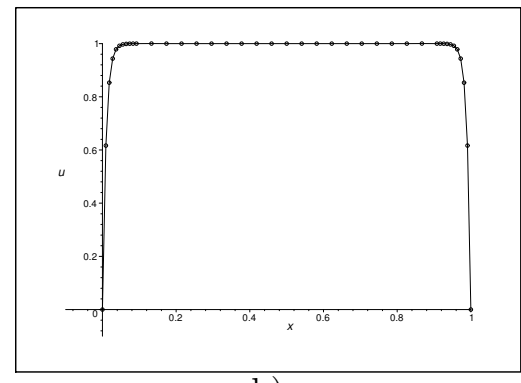

b)

Figure 1. The solution of problem (4.3) and apriori adaptive meshes: a) the Bakhvalov mesh for $\left.N=40, \varepsilon=0.05, \sigma=\frac{5}{2}, k=q=f=p=1, \mathrm{~b}\right)$ the Shishkin mesh for $N=40$, $\varepsilon=0.01, \sigma=\frac{5}{2}, k=q=f=p=1$.

from which $x_{j}$ is defined by:

$$
x_{j}=-\frac{\sigma \varepsilon}{\rho} \ln \left(1-\frac{2 j}{N}\left(1-e^{-\frac{\rho}{2 \sigma \varepsilon}}\right)\right), \quad j=1, \ldots, N / 2 .
$$

Since the mesh is symmetric with respect to point $x=0.5$, then $x_{j}=x_{N-j}$ for $j=N / 2+1, \ldots, N$.

The Bakhvalov mesh is exponentially fitted at boundary layers. In Figure 1a an example of the Bakhvalov mesh is shown for $f=q=k=p=1, \varepsilon=0.05$, discretization nodes are marked by vertical lines, $N=40$.

\subsection{Comparison with the Shishkin mesh}

In this subsection we investigate a relation between the Bakhvalov and Shishkin meshes. The Shishkin mesh is computed in two steps: first we define the thickness of boundary layers

$$
\lambda=\min \left(\frac{1}{4}, \frac{\sigma \varepsilon}{\rho} \ln N\right) .
$$

Next the interval $[0,1]$ is partitioned into three parts: $[0, \lambda),(\lambda, 1-\lambda),(1-\lambda, 1]$. Intervals $[0, \lambda)$ and $(1-\lambda, 1]$ are uniformly divided into $\frac{N}{4}$ parts, the interval $(\lambda, 1-\lambda)$ is uniformly divided into $\frac{N}{2}$ parts. An example of the Shishkin mesh is presented in Fig. 1b.

Our aim is to find a relation between formula (4.8), which defines the Bakhvalov mesh, and the Shishkin mesh. We restrict to the case when a mesh is still not very dense, then the parameter $\lambda$ is defined by

$$
\lambda=\frac{\sigma \varepsilon}{\rho} \ln N
$$

Since $e^{(-\sigma \varepsilon / \rho)} \ll 1$ and $N \gg 1$, then we get from (4.8) that 


$$
\begin{aligned}
x_{N / 2-1} & =-\frac{\sigma \varepsilon}{\rho} \ln \left(1-\frac{N-2}{N}\left(1-e^{-\frac{\rho}{2 \sigma \varepsilon}}\right)\right) \\
& \approx-\frac{\sigma \varepsilon}{\rho} \ln \left(1-\frac{N-2}{N}\right) \approx \frac{\sigma \varepsilon}{\rho} \ln N .
\end{aligned}
$$

Thus the Bakhvalov mesh gives a simple rule how to obtain the value of parameter $\lambda$ for the Shishkin mesh: take $\lambda=x_{N / 2-1}$.

We summarize main assumptions that were used to derive the Bakhvalov and Shishkin meshes from the adaptive mesh constructed by using dualitybased a posteriori estimates:

1. The apriori meshes do not depend on local values of $q(x)$, the global parameter $\rho=\min _{x \in[0,1]} \sqrt{q(x)}$ is used, i.e. both methods ignore the fact that the thickness of left and right boundary layers can be different.

2. The apriori meshes do not depend on local values of $f(x)$, i.e. both methods ignore the fact that the stiffness of boundary layers can be different.

3. Exponentially fitted distribution of nodes in the Bakhvalov mesh is changed to a piecewise uniform one in the Shishkin mesh.

We note that the convergence rate of standard finite difference schemes is $\mathcal{O}\left(N^{-2}\right)$ for the Bakhvalov mesh and $\mathcal{O}\left(N^{-2} \ln ^{2} N\right)$ for the Shishkin mesh. The difference in convergence rates is not essential, at the same time the Shishkin mesh is much simpler and it can be generalized for multidimensional problems with different types of boundary layers.

\subsection{Modifications of the Shishkin mesh}

In this section we modify the Shishkin mesh by relaxing the first two assumptions. Mackenzie and Beckett [6] have proposed to use different boundary layer thickness parameters for different boundaries. We also apply this rule. The thicknesses of boundary layers are defined by using local values function $q(x)$ :

$$
\lambda_{0}=\frac{\sigma \varepsilon}{\sqrt{q(0)}} \ln N, \quad \lambda_{1}=\frac{\sigma \varepsilon}{\sqrt{q(1)}} \ln N
$$

where $\sigma=\frac{2 p+1}{2}$. We divide the whole interval $[0,1]$ into three parts: $\left[0, \lambda_{0}\right)$, $\left(\lambda_{0}, \lambda_{1}\right)$ and $\left(\lambda_{1}, 1\right]$. A regular part $\left(\lambda_{0}, \lambda_{1}\right)$ is divided into $\frac{N}{2}$ intervals, and $\frac{N}{2}$ intervals are used to discretize boundary layers $\left(0, \lambda_{0}\right)$ and $\left(\lambda_{1}, 1\right)$.

Aposteriori error estimates (4.4) show that the source function $f(x)$ affects the residual error for different boundary layers. We use a simple rule how to define the numbers of mesh points in each boundary layer. By applying the mid-point integral approximation, Let us rewrite the equidistribution equation (3.10) as

$$
h_{j-0.5}\left|(q U-f)_{j-0.5}\right|^{1 / \sigma}=\text { const }, \quad j=1,2, \ldots, N,
$$

with $p=1,2$. By using the differential equation (1.1) we get the equivalent formulation, that the adaptive grid is generated by equidistributing the moni- 
toring functional

$$
M(u):=\int_{0}^{1}\left|u^{\prime \prime}(x)\right|^{1 / \sigma} d x, \quad \sigma=\frac{2 p+1}{2} .
$$

Substituting asymptotics of the solution at the boundary layers

$$
u(x)= \begin{cases}\frac{f(0)}{q(0)} e^{-\sqrt{q(0) / \varepsilon^{2}} x}, & 0 \leq x \leq \lambda_{0}, \\ \frac{f(1)}{q(1)} e^{-\sqrt{q(1) / \varepsilon^{2}}(1-x)}, & 1-\lambda_{1} \leq x \leq 1,\end{cases}
$$

into the monitoring functional (4.10), we get that the numbers of mesh points within each layer should be proportional to weights of the monitoring indicators

$$
W_{0}:=\int_{0}^{\lambda_{0}}\left|u^{\prime \prime}(x)\right|^{1 / \sigma} d x=c \frac{|f(0)|^{1 / \sigma}}{\sqrt{q(0)}}, \quad W_{1}:=\int_{1-\lambda_{1}}^{1}\left|u^{\prime \prime}(x)\right|^{1 / \sigma} d x=c \frac{|f(1)|^{1 / \sigma}}{\sqrt{q(1)}},
$$

where $c=\sigma \varepsilon^{1-2 / \sigma}(1-1 / N)$. Thus the numbers of mesh points within each layer are defined as (see, also [6]):

$$
N_{0}=\frac{N}{2} \frac{|f(0)|^{1 / \sigma} / \sqrt{q(0)}}{|f(0)|^{1 / \sigma} / \sqrt{q(0)}+|f(1)|^{1 / \sigma} / \sqrt{q(1)}}, \quad N_{1}=\frac{N}{2}-N_{0} .
$$

The uniform norm. The Shishkin mesh was developed for the numerical analysis of singular boundary problems in the uniform norm. The aposteriori error estimates are defined for the $L_{2}$ and energy norms. We propose the following heuristic algorithm, which is based on the error estimation of the piecewise linear interpolant $\pi_{h}$. Let us denote by $h_{0}=\frac{\lambda_{0}}{N_{0}}$ and $h_{1}=\frac{\lambda_{1}}{N_{1}}$. Consider the left-hand layer in detail. Simple computations prove that

$$
\begin{aligned}
& \max _{0 \leq x \leq \lambda_{0}}\left|\left(I-\pi_{h}\right)\left(\frac{f(0)}{q(0)} e^{-\sqrt{q(0) / \varepsilon^{2}} x}\right)\right| \\
& =\frac{|f(0)|}{q(0)} \frac{h_{0}^{2}}{4}\left|\left(e^{-\sqrt{q(0) / \varepsilon^{2}} x}\right)^{\prime \prime}\right|_{x=\frac{h_{0}}{2}} \mid+\mathcal{O}\left(h^{4}\right) \\
& =\frac{h_{0}^{2}}{4 \varepsilon^{2}}|f(0)| e^{-\sqrt{q(0) / \varepsilon^{2}} h_{0} / 2}+\mathcal{O}\left(h^{4}\right)=\frac{h_{0}^{2}}{4 \varepsilon^{2}}|f(0)|+\mathcal{O}\left(h^{3}\right) .
\end{aligned}
$$

For the right-hand layer we get similarly that

$$
\max _{1-\lambda_{1} \leq x \leq 1}\left|\left(I-\pi_{h}\right)\left(\frac{f(1)}{q(1)} e^{-\sqrt{q(1) / \varepsilon^{2}}(1-x)}\right)\right|=\frac{h_{1}^{2}}{4 \varepsilon^{2}}|f(1)|+\mathcal{O}\left(h^{3}\right) .
$$

Thus the numbers of mesh points within the different layers should satisfy the following requirement

$$
\frac{N_{1}}{N_{0}}=\frac{\sqrt{f(1)} \lambda_{1}}{\sqrt{f(0)} \lambda_{0}}=\frac{\sqrt{f(1) / q(1)}}{\sqrt{f(0) / q(0)}} .
$$


By using the equality $N_{0}+N_{1}=N / 2$ we get

$$
N_{0}=\frac{N}{2} \frac{\sqrt{f(0) / q(0)}}{\sqrt{f(0) / q(0)}+\sqrt{f(1) / q(1)}}, \quad N_{1}=\frac{N}{2}-N_{0} .
$$

Comparing (4.12) with (4.11), we see that for the uniform norm the distribution of mesh nodes is defined by the same formula but with the parameter $\sigma=2$.

In order to guarantee that mesh steps in boundary layers are not larger than in a regular area, we formulate a modification of the Shishkin algorithm:

1. Calculate thicknesses of boundary layers

$$
\lambda_{0}=\min \left(0.25, \frac{\sigma \varepsilon}{\sqrt{q(0)}} \ln N\right), \quad \lambda_{1}=\min \left(0.25, \frac{\sigma \varepsilon}{\sqrt{q(1)}} \ln N\right)
$$

2. Calculate from (4.12) the numbers of mesh nodes in boundary layers.

3. Regularize the mesh:

a) If $h_{1 / 2}>h_{N_{0}+1 / 2}$, then $N_{0}=\lambda_{0} / h_{N_{0}+1 / 2}, N_{1}=N / 2-N_{0}$;

b) If $h_{N-1 / 2}>h_{N_{0}+1 / 2}$, then $N_{1}=\lambda_{1} / h_{N_{0}+1 / 2}, N_{0}=N / 2-N_{1}$.

4. Intervals $\left[0, \lambda_{0}\right),\left(\lambda_{0}, \lambda_{1}\right)$ and $\left(\lambda_{1}, 1\right]$ are uniformly divided into $N_{0}, N / 2$ and $N_{1}$ parts, respectively.

\subsection{Numerical experiments}

In this section we investigate how assumptions used to construct the Shishkin mesh affect the accuracy of the discrete solution. For error computations we have computed numerical solutions by taking a very big number of mesh points $N=16000$ of the Bakhvalov mesh, the mesh regularization parameter $\alpha$ in formula (4.2) is defined by the technique offered in Beckett and Mackenzie [6]. In all computations we use $\sigma=\frac{5}{2}$. Computational experiments are done for the Shishkin mesh and adaptive mesh based on a posteriori error estimate in the $L_{2}$ norm. Nodal errors $e(x)=u(x)-U(x)$ in the maximum norm are also presented.

Example 1. Consider the following singular problem

$$
\left\{\begin{array}{l}
-\varepsilon^{2} u^{\prime \prime}+u=x, \quad \text { in } x \in(0,1) \\
u(0)=0, \quad u(1)=0
\end{array}\right.
$$

The solution of this problem do not have boundary layer singularity on the left side of interval. The adaptive mesh based on a posteriori error estimate, the Galerkin solution and global errors of this solution are presented in Fig. 2. The most of mesh points are distributed on the right boundary layer at $x=1$ and they are adapted to local values of $f$.

At it was stated above, the Shishkin mesh does not depend on local values of the function $f(x)$ and the mesh points are adapted to both boundary layers, 


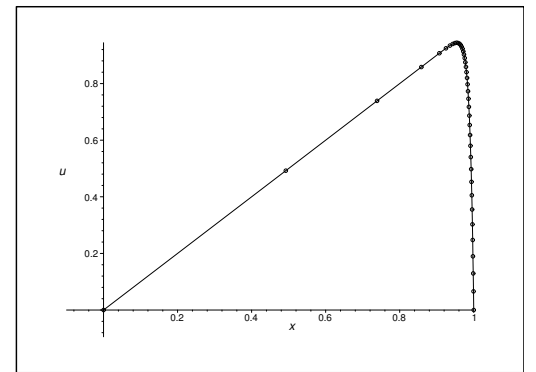

a)

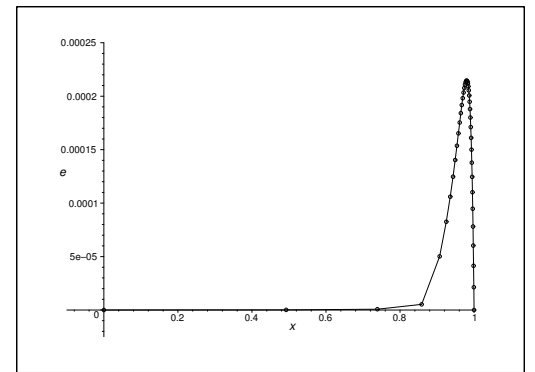

b)

Figure 2. Results for Example 1 with $\varepsilon=0.01$ : a) an adaptive mesh based on a posteriori error estimates and the Galerkin solution, b) the global error $|e(x)|$ for problem (4.13).

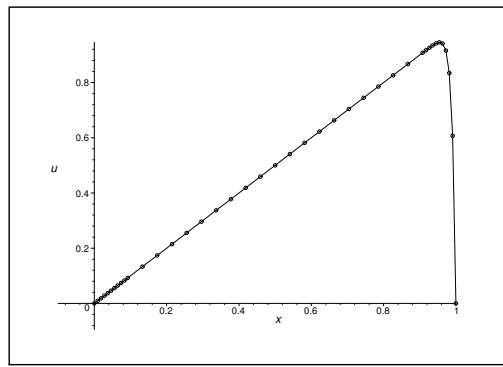

a)

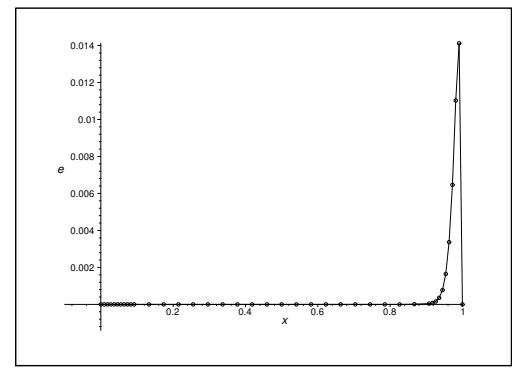

b)

Figure 3. Results for Example 1 with $\varepsilon=0.01$ : a) the Shishkin mesh and the Galerkin solution, b) the global error $|e(x)|$ for problem (4.13).

Table 1. Errors of the Galerkin solution in the $L_{2}$ norm and the experimental convergence rates for problem (4.13).

\begin{tabular}{lcccc}
\hline$n$ & the Shishkin mesh & rate & adaptive mesh & rate \\
\hline 20 & $1.08 \mathrm{E}-2$ & - & $1.81 \mathrm{E}-4$ & - \\
40 & $4.36 \mathrm{E}-3$ & 1.31 & $3.78 \mathrm{E}-5$ & 2.26 \\
80 & $1.58 \mathrm{E}-3$ & 1.46 & $8.70 \mathrm{E}-6$ & 2.12 \\
160 & $5.36 \mathrm{E}-4$ & 1.56 & $2.09 \mathrm{E}-6$ & 2.06 \\
320 & $1.74 \mathrm{E}-4$ & 1.63 & $5.11 \mathrm{E}-7$ & 2.03 \\
640 & $5.45 \mathrm{E}-5$ & 1.67 & $1.26 \mathrm{E}-7$ & 2.02 \\
\hline
\end{tabular}

though the left one is not presented in this example. We see in Fig. 3, that for the Shishkin mesh the largest errors of the Galerkin solution are distributed near the right boundary layer.

Next we have solved problem (4.13) and computed the error by using both meshes with different numbers of nodes $N$. The errors in the $L_{2}$ norm and the experimental convergence rates are given in Table 1.

It follows from the presented results, that the adaptive mesh based on a posteriori error estimates gives more accurate Galerkin solution than the Shishkin mesh. However the difference between both results is not big. 


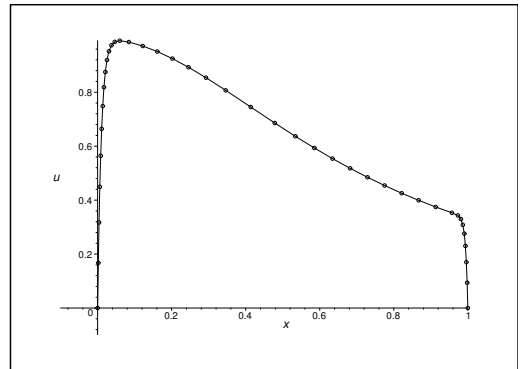

a)

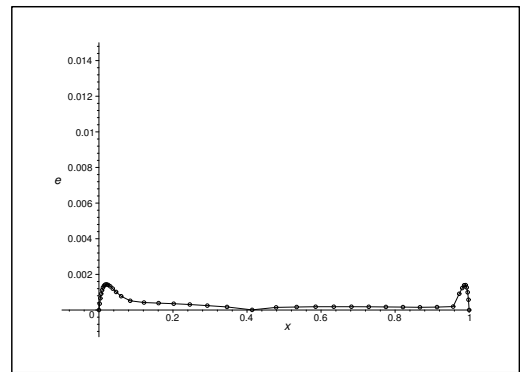

b)

Figure 4. Results for Example 2: a) an adaptive mesh based on a posteriori error estimates and the Galerkin solution, b) the global error $|e(x)|$ for problem (4.14).

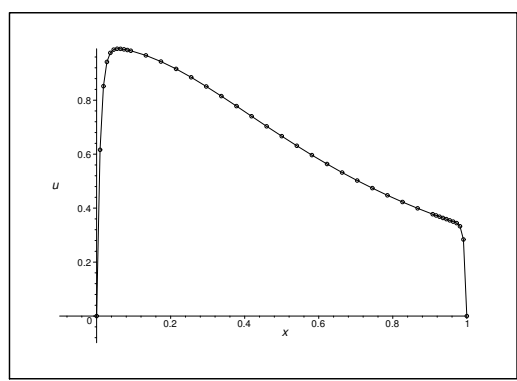

a)

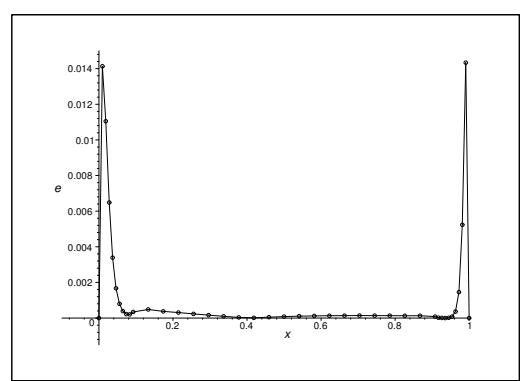

b)

Figure 5. Results for Example 2: a) the Shishkin mesh and the Galerkin solution, b) the global error $|e(x)|$ for problem (4.14).

Example 2. In this example we consider a singular problem with different thicknesses of boundary layers:

$$
\left\{\begin{array}{l}
-\varepsilon^{2} u^{\prime \prime}+\left(1+2 x^{2}\right) u=1, \quad \text { in } x \in(0,1), \\
u(0)=0, \quad u(1)=0 .
\end{array}\right.
$$

The thickness of the right boundary layer is smaller than the thickness of the left boundary layer. The adaptive mesh based on a posteriori error estimate, the Galerkin solution and global errors of this solution are presented in Fig. 4. We see that global error of the Galerkin solution is well balanced at both boundary layers.

The Shishkin mesh does not depend on local values of the function $q(x)$, thus the thickness of both boundary layers is taken the same in this case. We see in Fig. 5, that for the Shishkin mesh the largest errors of the Galerkin solution are distributed near the right boundary layer, where the real thickness is smaller than defined by the mesh and some mesh points are redundant there.

Next we have solved problem (4.14) and computed the error by using adaptive and the Shishkin meshes with different numbers of nodes $N$. The errors in the $L_{2}$ norm and the experimental convergence rates are given in Table 2. 
Table 2. Errors of the Galerkin solution in the $L_{2}$ norm and the experimental convergence rates for problem (4.14).

\begin{tabular}{lcccc}
\hline$n$ & the Shishkin mesh & rate & adaptive mesh & rate \\
\hline 20 & $1.28 \mathrm{E}-2$ & - & $1.82 \mathrm{E}-3$ & - \\
40 & $5.33 \mathrm{E}-3$ & $1.27 \mathrm{E}$ & $3.31 \mathrm{E}-4$ & $2.46 \mathrm{E}$ \\
80 & $1.96 \mathrm{E}-3$ & $1.44 \mathrm{E}$ & $7.02 \mathrm{E}-5$ & $2.24 \mathrm{E}$ \\
160 & $6.70 \mathrm{E}-4$ & $1.55 \mathrm{E}$ & $1.66 \mathrm{E}-5$ & $2.08 \mathrm{E}$ \\
320 & $2.18 \mathrm{E}-4$ & $1.62 \mathrm{E}$ & $4.14 \mathrm{E}-6$ & $2.01 \mathrm{E}$ \\
640 & $6.84 \mathrm{E}-5$ & $1.67 \mathrm{E}$ & $1.04 \mathrm{E}-6$ & $2.00 \mathrm{E}$ \\
\hline
\end{tabular}

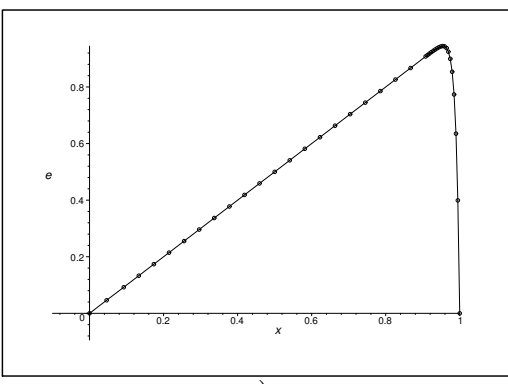

a)

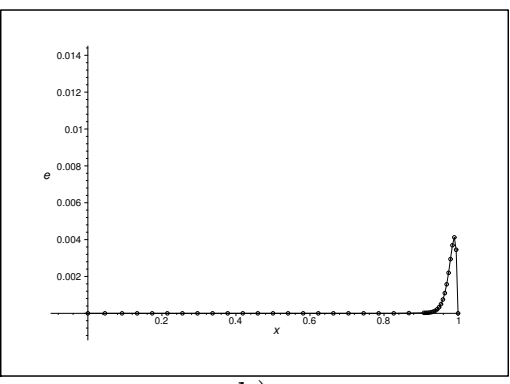

b)

Figure 6. Results for Example 1: a) the modificated Shishkin mesh and the Galerkin solution, b) the global error $|e(x)|$ for problem (4.13).

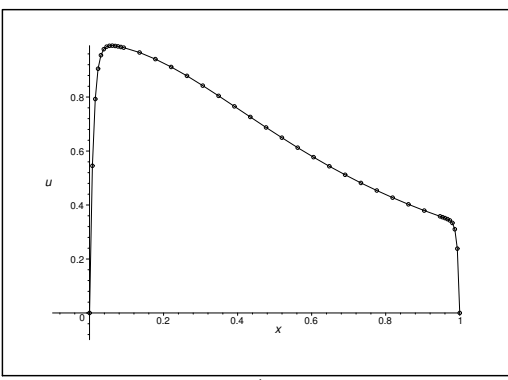

a)

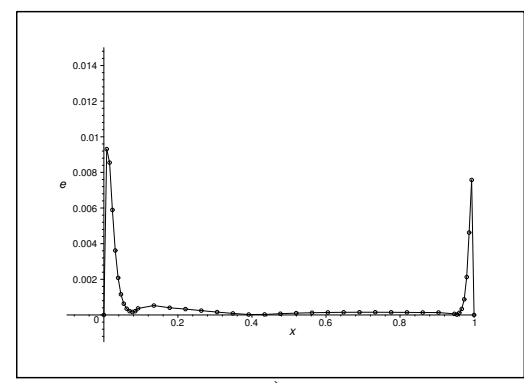

b)

Figure 7. Results for Example 2: a) the modificated Shishkin mesh and the Galerkin solution, b) the global error $|e(x)|$ for problem (4.14).

We have solved the same test problems by using the modified Shishkin mesh. Results are presented in Fig. 6 for problem (4.13) and in Fig. 7 for problem (4.14). Comparing these results with results obtained using the classical Shishkin mesh (see, Figs. 3 and 5), we conclude that in both cases the distribution of nodes with the modified Shishkin mesh gives smaller errors.

Also, we have solved problem (4.13) with a smaller parameter $\varepsilon=10^{-3}$ and computed the error by using the classical and modified Shishkin meshes with different numbers of nodes $N$. The errors in the $L_{2}$ norm and the experimental convergence rates are given in Table 3 . 
Table 3. Errors of the Galerkin solution in the $L_{2}$ norm and the experimental convergence rates for problem $(4.14), \varepsilon=10^{-3}$.

\begin{tabular}{lcccc}
\hline$n$ & the Shishkin mesh & rate & the modified Shishkin mesh & rate \\
\hline 20 & $3.42 \mathrm{E}-3$ & - & $9.23 \mathrm{E}-4$ & - \\
40 & $1.38 \mathrm{E}-3$ & 1.31 & $3.56 \mathrm{E}-4$ & 1.37 \\
80 & $5.00 \mathrm{E}-4$ & 1.46 & $1.26 \mathrm{E}-4$ & 1.49 \\
160 & $1.69 \mathrm{E}-4$ & 1.56 & $4.36 \mathrm{E}-5$ & 1.54 \\
320 & $5.49 \mathrm{E}-5$ & 1.63 & $1.41 \mathrm{E}-5$ & 1.63 \\
640 & $1.72 \mathrm{E}-5$ & 1.67 & $4.45 \mathrm{E}-6$ & 1.66 \\
\hline
\end{tabular}

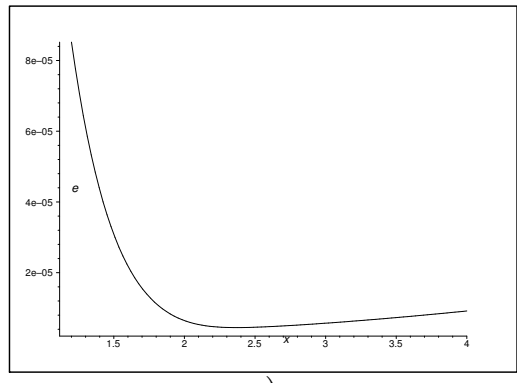

a)

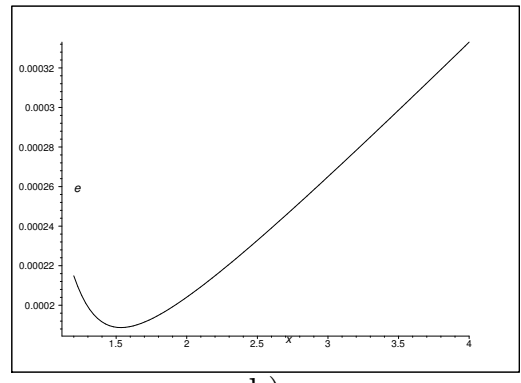

b)

Figure 8. The dependence of global errors on parameter $\sigma$ : a) the $L_{2}$ norm, b) the energy norm.

From Table 3 we see that Galerkin solutions for both meshes have similar rates of convergence. But absolute values of errors differs four times, that means that using the classical Shishkin mesh we must take two times more nodes than for the modified Shishkin mesh in order to achieve the same accuracy of the solution. Similar results are obtained for problem (4.14).

Optimal values of parameter $\sigma$. In Section 4 we have derived optimal values of parameter $\sigma$ for the apriori adaptive Bakhvalov and Shishkin meshes, i.e. $\sigma=\frac{5}{2}$ should be used for the global error control in the $L_{2}$ norm and $\sigma=\frac{3}{2}$ is optimal for the energy norm.

Next we present results of numerical experiments, where the optimality of these parameters is investigated Let us consider a singular problem

$$
\left\{\begin{array}{l}
-10^{-8} u^{\prime \prime}+u=1, \quad \text { in } x \in(0,1) \\
u(0)=0, \quad u(1)=0
\end{array}\right.
$$

We use the Bakhvalov mesh with $N=160$ nodes and $\alpha=0$.

In Figure 8a we show the dependence of the error $\|e\|$ on the mesh parameter $\sigma$, and in b part of this figure the dependence of the error $\left\|e^{\prime}\right\|$ is shown.

It follows from the presented results that experimental optimal values $\sigma=$ 2.37 and $\sigma=1.54$ are close to theoretical values $\sigma=2.5$ and $\sigma=1.5$ obtained from adaptive meshes based on a posteriori error estimates. 


\section{Conclusions}

- The Bakhvalov and Shishkin meshes were derived from a posteriori duality based error estimations.

- We have derived a relation between a norm in which the error is controlled and optimal values of parameter $\sigma$ which is used in definition of the Bakhvalov and Shishkin meshes.

- The modification of the Shishkin mesh is proposed and investigated.

\section{Acknowledgements}

Authors thank Prof. G. Shishkin for useful discussions and referees of the paper for suggestions that lead to improvement of the presentation.

\section{References}

[1] G. Amiraliyev and M. Cakir. Numerical solution of singularly perturbed problem with nonlocal boundary condition. Appl. Math. Mech., 23(7):755-764, 2002. http://dx.doi.org/10.1007/BF02456971.

[2] L. Angermann. Balanced aposteriori error estimates for finite volume type discretizations of convection-dominated elliptic problems. Computing, 55:305-323, 1995. http://dx.doi.org/10.1007/BF02238485.

[3] Th. Apel and G. Lube. Anisotropic mesh refinement for a singularly perturbed reaction diffusion model problem. Appl. Numer. Math., 26:415-433, 1998. http://dx.doi.org/10.1016/S0168-9274(97)00106-2.

[4] N.S. Bakhvalov. Towards optimization of methods for solving boundary value problems in the presence of boundary layers. Zh. Vychisl. Mat. Mat. Fiz., 9:841859, 1969.

[5] W. Bangerth and R. Rannacher. Adaptive Finite Element Methods for Differential Equations. Birkhäuser Verlag, 2003.

[6] G. Beckett and J. Mackenzie. On a uniformly accurate finite-difference approximation of a singularly perturbed reaction-diffusion problem using grid equidistribution. J. Comput. Appl. Math., 131:381-405, 2001.

http://dx.doi.org/10.1016/S0377-0427(00)00260-0.

[7] I.P. Boglaev. The numerical solution of a nonlinear boundary value problem with small parameter effecting the highest derivative. Zh. Vychisl. Mat. Mat. Fiz., 24:1649-1656, 1984.

[8] M. Cakir and G. Amiraliyev. A finite difference method for the singularly perturbed problem with nonlocal boundary condition. Appl. Math. Comput., 160:539-549, 2005. http://dx.doi.org/10.1016/j.amc.2003.11.035.

[9] R. Čiegis. Numerical solution of a problem with small parameter for the highest derivative and a nonlocal condition. Lithuanian Math. J., 28(1):90-96, 1988. http://dx.doi.org/10.1007/BF00972255.

[10] K. Eriksson, D. Estep, P. Hansbo and C. Johnson. Introduction to adaptive methods for differential equations. Acta Numer., 5:105-158, 1995.

http://dx.doi.org/10.1017/S0962492900002531. 
[11] A.M. Il'in. A difference scheme for a differential equation with a small parameter affecting the highest derivative. Mat. Zametki, 6:237-248, 1969.

[12] V. John. A numerical study of a posteriori error estimators for convectiondiffusion equations. Comput. Methods Appl. Mech. Engrg., 190:757-781, 2000. http://dx.doi.org/10.1016/S0045-7825(99)00440-5.

[13] M. Kadalbajoo and V. Gupta. A brief survey on numerical methods for solving singularly perturbed problems. Appl. Math. Comput., 10(3):3641-3716, 2010. http://dx.doi.org/10.1016/j.amc.2010.09.059.

[14] G. Kunert. A posteriori $H^{1}$ error estimation for a singularly perturbed reactiondiffusion problem on anisotropic meshes. IMA J. Numer. Anal., 25(2):408-428, 2005. http://dx.doi.org/10.1093/imanum/drh009.

[15] T. Linß. Maximum-norm error analysis of a non-monotone FEM for a singularly perturbed reaction-diffusion problem. BIT Numer. Math., 47:379-391, 2007.

[16] J. Mackenzie. Uniform convergence analysis of an upwind finite-difference approximation of a convection-diffusion boundary value problem on an adaptive grids. IMA J. Numer. Anal., 19(2):233-249, 1999.

http://dx.doi.org/10.1093/imanum/19.2.233.

[17] Y. Qiu and D. Sloan. Analysis of difference approximations to a singularly perturbed two-point boundary value problem on an adaptively generated grid.

J. Comput. Appl. Math., 101:1-25, 1999.

http://dx.doi.org/10.1016/S0377-0427(98)00136-8.

[18] H. Roos and T. Linß. Sufficient conditions for uniform convergence on layeradapted grids. Computing, 63:27-45, 1999.

http://dx.doi.org/10.1007/s006070050049.

[19] H. Roos, M. Stynes and L. Tobiska. Numerical Methods for Singularly Perturbed Differential Equations. Springer, Berlin, 1996.

[20] A.A. Samarskii. The Theory of Difference Schemes. Marcel Dekker, Inc., New York-Basel, 2001.

[21] G. Shishkin. Grid Approximation of Singularly Perturbed Elliptic and Parabolic Equations. PhD thesis, Keldysh Institute, Moscow, 1990.

[22] G. Stoyan. Explicit error estimates for difference schemes solving the stationary constant coefficient diffusion-convection-reaction equation. Z. Angew. Math. Mech., 64:173-191, 1984. http://dx.doi.org/10.1002/zamm.19840640306.

[23] M. Stynes and E. O'Riordan. A finite element method for a singularly perturbed boundary value problem. Numer. Math., 50(1):1-15, 1986. http://dx.doi.org/10.1007/BF01389664.

[24] R. Vulanovic. Mesh Construction for Discretization of Singularly Perturbed Boundary Value Problems. PhD thesis, University of Novi Sad, Novi Sad, 1986.

[25] R. Vulanovic. A priori meshes for singularly perturbed quasilinear two-point boundary value problems. IMA J. Numer. Anal., 21(1):349-366, 2001. http://dx.doi.org/10.1093/imanum/21.1.349.

[26] R. Vulanovic. The layer-resolving transformation and mesh generation for quasilinear singular perturbation problems. J. Comput. Appl. Math., 203:177-189, 2007. http://dx.doi.org/10.1016/j.cam.2006.03.020. 